\title{
Accuracy of Intraocular Lens Power Calculation Formulas in Myopic Eyes with Target Refractions of Emmetropia and Intentional Myopia
}

\author{
Daiki Sakai (iD ${ }^{1-3}$ \\ Yasuhiko Hirami ${ }^{1,2}$ \\ Makoto Nakamura $\mathbb{D}^{3}$ \\ Yasuo Kurimoto ${ }^{1,2}$ \\ 'Department of Ophthalmology, Kobe \\ City Eye Hospital, Kobe, Japan; \\ ${ }^{2}$ Department of Ophthalmology, Kobe \\ City Medical Center General Hospital, \\ Kobe, Japan; ${ }^{3}$ Department of Surgery, \\ Division of Ophthalmology, Kobe \\ University Graduate School of Medicine, \\ Kobe, Japan
}

Correspondence: Daiki Sakai Department of Ophthalmology, Kobe City Eye Hospital, 2-I-8 Minatojima Minamimachi, Chuo-ku, Kobe-shi, Hyogo, 650-0047, Japan

Tel $+8 \mathrm{I}-78-38 \mathrm{I}-9876$

$\mathrm{Fax}+81-78-381-9910$

Email dsakail027@gmail.com
Purpose: To compare the accuracy of the intraocular lens (IOL) power calculation formulas for predicting the postoperative refraction in eyes with a target of emmetropia or intentional myopia.

Patients and Methods: This is a retrospective study conducted at Kobe City Eye Hospital, Kobe, Japan. Fifty eyes of 50 patients with axial myopia who underwent uncomplicated phacoemulsification and single-type IOL implantation for a target of emmetropia (plano to $-0.5 \mathrm{D})$ or intentional myopia $(-2.0 \mathrm{D}$ to $-3.0 \mathrm{D})$ were selected. Preoperative ocular biometry was performed using IOLMaster700 in all eyes. Refractive prediction errors of 6 IOL formulas integrated into IOLMaster700 were compared between eyes with a target of emmetropia and intentional myopia.

Results: The mean numerical errors of SRK/T (Sanders, Retzlaff, and Kraft/theoretical), Holladay 1, Hoffer Q, and Holladay 2 significantly differed between the two groups ( $\mathrm{p}<$ $0.001, p=0.008,0.02$, and 0.007 , respectively). The values for mean numerical errors in eyes with a target of intentional myopia were smaller, showing relatively myopic outcome, as compared with those in eyes with a target of emmetropia. In eyes with a target of emmetropia, the mean numerical errors of Holladay $1(\mathrm{p}<0.001,95 \%$ confidence interval $[\mathrm{CI}]$ : 0.32 to 0.63 ), Hoffer Q ( $\mathrm{p}=0.001,95 \%$ CI: 0.12 to 0.42 ), and Barrett Universal II ( $\mathrm{p}=$ $0.007,95 \% \mathrm{CI}: 0.06$ to 0.35 ) were significantly different from zero (hyperopic trend). Furthermore, in eyes with a target of intentional myopia, the mean numerical error of SRK $/ \mathrm{T}(\mathrm{p}=0.001,95 \% \mathrm{CI}:-0.61$ to -0.17$)$ and Holladay $2(\mathrm{p}=0.023,95 \% \mathrm{CI}:-0.43$ to -0.04 ) were significantly different from zero (myopic trend).

Conclusion: In patients with axial myopia, some IOL formulas may show a myopic trend in the refractive outcome when targeting intentional myopia as compared to emmetropia.

Keywords: cataract surgery, postoperative refraction, intentional myopia, axial myopia, IOLMaster700

\section{Introduction}

Modern cataract surgery has evolved to include a refractive procedure with improvements in ocular biometry and intraocular lens (IOL) power calculation formula. Patients undergoing cataract surgery have an opportunity to achieve a preferred postoperative refraction. Patients who are originally myopic often want to retain myopia to maintain good vision at near-distance. Therefore, targeting intentional myopia is an option in addition to targeting emmetropia in patients with myopia. For targeting intentional myopia, a target refraction of $-2.0 \mathrm{D}$ or $-3.0 \mathrm{D}$ is generally acceptable. ${ }^{1,2}$ The IOL power calculation formulas are considered to 
develop for achieving emmetropia after cataract surgery, and previous reports have mainly assessed their accuracy when targeting emmetropia. However, an important concern that has been suggested in few reports is that the IOL power calculation formulas might be less accurate for targeting myopia as compared to emmetropia. ${ }^{3,4}$ So far, there is limited research done on this topic. The purpose of this study was to compare the accuracy of IOL power calculation formulas for predicting the postoperative refraction in eyes with a target of emmetropia and intentional myopia.

\section{Methods}

This retrospective study was performed in accordance with the Declaration of Helsinki and was approved by the Medical Ethics Committee of the Kobe City Medical Center General Hospital (Kobe, Japan). We applied an optout arrangement to obtain the informed consent for this observational study, which involved the analysis of medical records. The confidentiality of patient data was maintained.

We reviewed the medical records of patients who underwent uncomplicated phacoemulsification, and implantation of a DCB00V (AMO, Santa Ana, CA, USA) IOL. The inclusion criteria were as follows: (1) originally myopic (2) axial length (AL) between 24.5 and $26.0 \mathrm{~mm}$; (3) target refraction was emmetropia (between 0 and $-0.50 \mathrm{D}$ ) or intentional myopia (between -2.00 and $-3.00 \mathrm{D}$ ). Exclusion criteria included a history of corneal diseases (pterygium, $\mathrm{n}=2$; keratoconus, $\mathrm{n}=1$ ), previous ocular surgery (trabeculectomy, $\mathrm{n}=4$; scleral buckling, $\mathrm{n}=1$ ), poor-quality ocular biometry ( $\mathrm{n}$ $=2)$, lack of postoperative data $(\mathrm{n}=2)$, postoperative refraction data collected within 2 weeks of surgery $(\mathrm{n}=$ 28 ), and a postoperative corrected distance visual acuity worse than 20/40 $(n=0)$. Additionally, if both eyes of one patient were eligible (20 patients), one eye was randomly selected taking into account the correlation between the eyes. ${ }^{5}$ Overall in our study, 26 eyes of 26 patients with a target of emmetropia, and 24 eyes of 24 patients with a target of intentional myopia were included.

Preoperative ocular biometry was performed using the IOLMaster 700 (Carl Zeiss Meditec, Jena, Germany). All surgeries were performed by one of the eight surgeons at the Kobe City Eye Hospital, between January 2020 and March 2021. The manifest refraction at the follow-up visit between 2 weeks and 3 months was recorded as the postoperative refraction. In case of multiple visits, the latest value was selected. We used Japanese standard visual acuity chart presented at five meters distance in order to obtain the manifest refraction. Prior to statistical analyses, the manifest refraction was adjusted to six meters, as suggested by Simpson and Charman. ${ }^{6}$ The predicted refraction values of the implanted IOL, calculated by integrating formulas (SRK/T [Sanders, Retzlaff, and Kraft/theoretical], Holladay 1, Hoffer Q, Holladay 2, Haigis, Barret Universal II) into the IOLMaster 700, were retrospectively recorded. The ULIB-optimized (User Group for Laser Interference Biometry) IOL constant was used. In one eye with a target of emmetropia, the predicted refraction with Holladay 2 was unavailable due to insufficient data. The prediction error was calculated by subtracting the predicted refraction value from the postoperative refraction value (numerical error), and the absolute error was defined as the absolute value of the numerical error. A negative numerical error indicated a myopic error, and a positive numerical error indicated a hyperopic error. The prediction error of each formula was compared between the eyes with refractive target of emmetropia and the eyes with target of intentional myopia. In addition, the prediction errors of the six calculation formulas for intentional myopia were compared. All statistical analyses were performed using SPSS version 25 for Windows (SPSS Inc, Chicago, IL, USA). The categorical variables were compared using Chi-square test, and the continuous variables were compared using unpaired $t$-test. One-sample t-tests were used to determine whether the numerical errors were statistically significantly different from zero for both groups (with targets of emmetropia and intentional myopia). Absolute errors between the six IOL calculation formulas was compared using repeated measures analysis of variance (ANOVA). Statistical significance of all tests was set at $\mathrm{p} \leq 0.05$.

In this study, eyes with high myopia ( $\mathrm{AL}>26.0 \mathrm{~mm})$ were excluded from the main analyses due to the reduced accuracy of IOL calculation formulas reported for such cases. ${ }^{7}$ These eyes were separately included in the study within secondary analyses. Inclusion and exclusion criteria for these secondary analyses were the same as for the main analyses except for the aforementioned $\mathrm{AL}$ requirement $(\mathrm{AL}>26.0 \mathrm{~mm})$. In total, 11 eyes of 11 patients with a target of emmetropia and 23 eyes of 23 patients with a target of intentional myopia were included in the secondary analyses. The prediction errors of the six formulas 
Table I Characteristics of the Patients and Implanted IOLs

\begin{tabular}{|c|c|c|c|}
\hline & \multicolumn{2}{|c|}{ Target } & \multirow[t]{2}{*}{ p value } \\
\hline & Emmetropia $(n=26)$ & Intentional Myopia $(n=24)$ & \\
\hline Age (years), mean $\pm S D$ & $74.1 \pm 8.3$ & $69.8 \pm 9.9$ & 0.10 \\
\hline Female, n (\%) & $8(30.8 \%)$ & $10(41.7 \%)$ & 0.42 \\
\hline \multicolumn{4}{|l|}{ Axial length (mm) } \\
\hline Mean \pm SD & $25.15 \pm 0.43$ & $25.19 \pm 0.40$ & 0.76 \\
\hline Range & $24.56-26.00$ & $24.53-25.96$ & \\
\hline \multicolumn{4}{|c|}{ Average keratometry (D) } \\
\hline Mean \pm SD & $43.49 \pm 1.55$ & $44.24 \pm 1.14$ & 0.06 \\
\hline Range & $39.86-47.87$ & $42.65-47.19$ & \\
\hline \multicolumn{4}{|c|}{ Anterior chamber depth $(\mathrm{mm})$} \\
\hline Mean \pm SD & $3.32 \pm 0.30$ & $3.37 \pm 0.36$ & 0.57 \\
\hline Range & $2.69-4.02$ & $2.75-4.05$ & \\
\hline \multicolumn{4}{|l|}{ Lens thickness (mm) } \\
\hline Mean \pm SD & $4.37 \pm 0.47$ & $4.31 \pm 0.47$ & 0.66 \\
\hline Range & $3.30-5.18$ & $3.33-5.11$ & \\
\hline \multicolumn{4}{|c|}{ Central corneal thickness $(\mu \mathrm{m})$} \\
\hline Mean \pm SD & $551.61 \pm 30.75$ & $545.12 \pm 39.69$ & 0.52 \\
\hline Range & $503.00-620.00$ & $454.20-612.01$ & \\
\hline \multicolumn{4}{|l|}{ White to white $(\mathrm{mm})$} \\
\hline Mean \pm SD & $11.98 \pm 0.44$ & $11.92 \pm 0.26$ & 0.57 \\
\hline Range & $11.05-12.74$ & $11.20-12.43$ & \\
\hline \multicolumn{4}{|l|}{ IOL power (D) } \\
\hline Mean \pm SD & $16.6 \pm 2.6$ & $19.4 \pm 1.9$ & $<0.001 *$ \\
\hline Range & $9.0-22.0$ & $13.5-22.5$ & \\
\hline
\end{tabular}

Notes: *Significant at $\mathrm{p}<0.05$ (unpaired $t$-test or chi-square test).

Abbreviations: IOL, intraocular lens; SD, standard deviation.

were compared between the two groups in the same manner as described for the main analyses.

\section{Results}

Fifty eyes of 50 patients (female patients: 18) were included in the study. Mean ( \pm SD) age of these 50 patients was $72.1 \pm 9.2$ years. Among these patients, 26 eyes were targeted for emmetropia (between 0 and -0.50 D) and 24 eyes were targeted for intentional myopia (between -2.00 and $-3.00 \mathrm{D}$ ).

Table 1 shows the clinical characteristics of the patients and the implanted IOL in eyes with a target of emmetropia and intentional myopia. There were no significant differences in the demographic and biometric data between the two groups. The mean power of the implanted IOL was significantly smaller in eyes with a target of emmetropia than in eyes with a target of intentional myopia.
A box and whisker plot of the numerical error for each IOL calculation formula is shown in Figure 1, and a comparison of the mean numerical error of the $6 \mathrm{IOL}$ calculation formulas between the two groups is shown in Table 2. We found that the mean numerical errors of the SRK/T, Holladay 1, Hoffer Q, and Holladay 2 formulas statistically significantly differed between the two groups $(\mathrm{p}<0.001, \mathrm{p}=0.008, \mathrm{p}=0.02$, and $\mathrm{p}=0.007$, respectively). For these four formulas, the values for mean numerical errors in eyes with a target of intentional myopia were smaller, showing a relatively myopic outcome, as compared with eyes with a target of emmetropia. Moreover, in eyes with a target of emmetropia, the mean numerical errors of Holladay 1 ( $p<0.001,95 \%$ confidence interval [CI]: 0.32 to 0.63$)$, Hoffer Q ( $p=0.001,95 \% \mathrm{CI}$ : 0.12 to 0.42 ), and Barrett Universal II ( $\mathrm{p}=0.007,95 \% \mathrm{CI}$ : 0.06 to 0.35 ) were significantly different from zero 


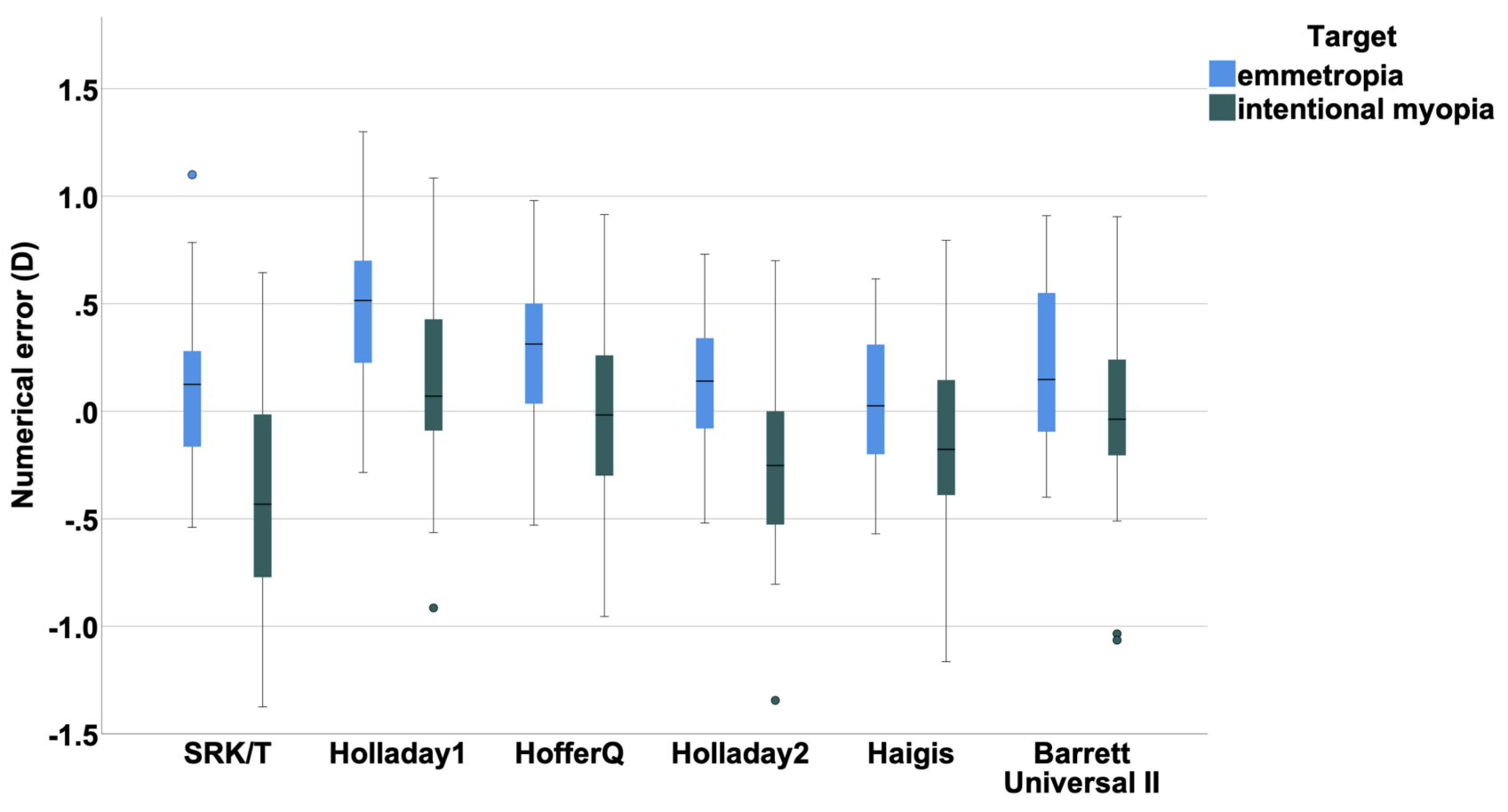

Figure I Box and whisker plots of numerical errors in eyes with a target of emmetropia and intentional myopia (SRK/T = Sanders, Retzlaff, and Kraft/theoretical). The horizontal line in the box represents the median. The top and bottom of the box represent the upper and lower quartiles, respectively. The whisker above the box represents the values within 1.5 times the interquartile range plus the upper quartile, and the whisker below the box represents the values within I.5 times the interquartile range minus the lower quartile. Values, which are not included between whiskers, were plotted as outliers.

(hyperopic trend). Lastly, the mean numerical error of $\mathrm{SRK} / \mathrm{T}(\mathrm{p}=0.001,95 \% \mathrm{CI}:-0.61$ to -0.17$)$ and Holladay 2 ( $p=0.023,95 \%$ CI: -0.43 to -0.04$)$ in eyes with a target of intentional myopia were significantly different from zero (myopic trend).

We also compared the mean absolute errors for the six IOL calculation formulas between the two groups (Table 3). The mean absolute error for the SRK/T formula in eyes with a target of intentional myopia was statistically significantly larger as compared with eyes with a target of emmetropia $(p=0.02)$. Overall, we found that there were no statistically significant differences in the mean absolute errors between the six IOL calculation formulas when targeting intentional myopia $(\mathrm{p}=0.39$ via repeated measures ANOVA).

The results of the secondary analyses that included eyes with high myopia (AL $>26.0 \mathrm{~mm}$ ) are presented in the supplemental materials (Supplemental Tables 1- $\underline{3}$ and Supplemental Figure 1). For all formulas, the values of mean numerical errors in eyes with a target of intentional myopia were comparatively reduced, showing a relatively myopic outcome as compared with the values in eyes with a target of emmetropia, although these differences were not statistically significant.

\section{Discussion}

This study compared the performance of 6 IOL power calculation formulas, among two groups of eyes with different postoperative refractive targets: emmetropia or intentional myopia. The results suggest that the refractive outcomes from some of the IOL power calculation formulas were different among eyes with a target of emmetropia and intentional myopia. The mean numerical errors of SRK/T, Holladay 1, Hoffer Q, and Holladay 2 were statistically significantly more myopic in the eyes targeted for myopia as compared to emmetropia. The mean numerical errors of Haigis and Barret Universal II were also more myopic in the eyes targeted for myopia as compared to emmetropia, although these differences were not statistically significant. Consequently, there was a significant myopic trend in the refractive outcome using SRK/T and Holladay 2 in eyes targeted for intentional myopia. Moreover, the significant hyperopic trend of the refractive outcomes using Holladay 1, Hoffer Q and Barret Universal II in eyes targeted for emmetropia was not found in eyes targeted for intentional myopia.

Intentional myopia is an option for target refraction after cataract surgery. In a previous report, about $10 \%$ of eyes were planned for myopia in a large Swedish cataract 
Table 2 Comparison of the Mean Numerical Errors of the IOL Power Calculation Formulas Between Eyes with Target of Emmetropia and Intentional Myopia

\begin{tabular}{|c|c|c|c|}
\hline & \multicolumn{3}{|c|}{ Target } \\
\hline & Emmetropia $(n=26 * * *)$ & Intentional Myopia $(n=24)$ & $\mathrm{p}$ value \\
\hline \multicolumn{4}{|l|}{ SRK/T } \\
\hline Numerical error (D), mean \pm SD & $0.09 \pm 0.40$ & $-0.39 \pm 0.53 * *$ & $<0.00$ I* \\
\hline Myopic error (\%) & $10(38.5 \%)$ & $18(75.0 \%)$ & \\
\hline \multicolumn{4}{|l|}{ Holladay I } \\
\hline Numerical error $(D)$, mean \pm SD & $0.48 \pm 0.38 * *$ & $0.14 \pm 0.48$ & $0.008 *$ \\
\hline Myopic error (\%) & $4(15.4 \%)$ & $10(41.7 \%)$ & \\
\hline \multicolumn{4}{|l|}{ Hoffer Q } \\
\hline Numerical error $(D)$, mean $\pm S D$ & $0.27 \pm 0.37 * *$ & $-0.02 \pm 0.48$ & $0.02 *$ \\
\hline Myopic error (\%) & $6(23.1 \%)$ & I 3 (54.2\%) & \\
\hline \multicolumn{4}{|l|}{ Holladay 2} \\
\hline Numerical error $(D)$, mean \pm SD & $0.11 \pm 0.38$ & $-0.23 \pm 0.47^{* *}$ & $0.007 *$ \\
\hline Myopic error (\%) & II (42.3\%) & I8 (75.0\%) & \\
\hline \multicolumn{4}{|l|}{ Haigis } \\
\hline Numerical error (D), mean \pm SD & $0.03 \pm 0.35$ & $-0.13 \pm 0.48$ & 0.17 \\
\hline Myopic error (\%) & $12(46.2 \%)$ & $15(62.5 \%)$ & \\
\hline \multicolumn{4}{|l|}{ Barrett Universal II } \\
\hline Numerical error $(D)$, mean $\pm S D$ & $0.21 \pm 0.36 * *$ & $-0.01 \pm 0.48$ & 0.08 \\
\hline Myopic error (\%) & $9(34.6 \%)$ & $13(54.2 \%)$ & \\
\hline
\end{tabular}

Notes: *Significant at $\mathrm{p}<0.05$ (unpaired $t$-test). $* *$ Significantly different from zero at $\mathrm{p}<0.05$ (one sample $t$-test). $* * * H o l l a d a y ~ 2: \mathrm{n}=25$.

Abbreviations: SRK/T, Sanders, Retzlaff, and Kraft/theoretical; SD, standard deviation; Myopic error, Postoperative refraction is relatively myopic compared to predicted refraction.

Table 3 Comparison of the Mean Absolute Errors of the IOL Power Calculation Formulas Between Eyes with Target of Emmetropia and Intentional Myopia

\begin{tabular}{|l|c|c|c|}
\hline \multirow{2}{*}{ Absolute Error (D), Mean \pm SD } & \multicolumn{3}{|c|}{ Target } \\
\cline { 2 - 4 } & Emmetropia (n= 26**) & Intentional Myopia (n = 24) & p value \\
\hline SRK/T & $0.31 \pm 0.26$ & $0.54 \pm 0.37$ & $0.02 *$ \\
Holladay I & $0.51 \pm 0.33$ & $0.37 \pm 0.33$ & 0.13 \\
Hoffer Q & $0.38 \pm 0.26$ & $0.37 \pm 0.29$ & 0.97 \\
Holladay 2 & $0.31 \pm 0.23$ & $0.40 \pm 0.32$ & 0.26 \\
Haigis & $0.29 \pm 0.18$ & $0.39 \pm 0.30$ & 0.18 \\
Barrett Universal II & $0.33 \pm 0.24$ & $0.35 \pm 0.32$ & 0.81 \\
\hline
\end{tabular}

Notes: *Significant at $\mathrm{p}<0.05$ (unpaired $t$-test). **Holladay 2: $\mathrm{n}=25$.

Abbreviations: SRK/T, Sanders, Retzlaff, and Kraft/theoretical; SD, standard deviation.

cohort study that included over 17,000 eyes. ${ }^{8}$ Note that, in patients who originally had myopia, a Japanese cohort reported that a vast majority (about $85 \%$ ) were planned for myopia. ${ }^{9}$ It is well known that the prevalence of myopia is increasing worldwide, especially in Southeast Asia, including Japan. ${ }^{10,11}$ Therefore, considering the expected increase in the frequency of cases, the refractive outcome of intentional myopia should be closely examined.

Few reports thus far have studied the accuracy of IOL power calculation formulas when targeting myopia. Geggel et al reported that the Haigis formula was less accurate when targeting myopia $(-1.0 \mathrm{D})$ than when targeting emmetropia. ${ }^{12}$ Turnbull et al conducted a paired- 
eye study in patients with planned monovision and reported that the SRK/T, Holladay 1, Hoffer Q, Haigis, Barrett Universal II, and Hill-RBF version 2.0 (Hill-Radial Basis Function) formulas were less accurate when targeting myopia $(-1.25 \mathrm{D})$ as compared to targeting emmetropia. ${ }^{4}$ These reports are concerning and highlight that the performance of the IOL power calculation formulas may be altered when targeting myopia. In this study, we found that some formulas may show a relative myopic trend in the refractive outcome when targeting myopia as compared to emmetropia. Our results are comparable to those of a previous report, where the Swedish cataract cohort study reported that eyes planned for myopia ( $\leq$ -1.6 D) became more myopic than initially predicted. ${ }^{8}$ However, the IOL power calculation formula was not registered as a variable in this study. Recently, Cooke et al also reported a myopic trend in the refractive outcome using SRK $/ T$ (mean numerical error $=-0.24 \mathrm{D}$ ) for intentional myopia $(\leq-1.75 \mathrm{D}),{ }^{13}$ which is consistent with our results. We also found a myopic trend in the refractive outcome using the Holladay2 formula when targeting intentional myopia, which had not been reported previously. Thus, possible changes in the performance of the IOL power calculation formulas should be kept in mind when using specific formulas for intentional myopia. Although we did not find any significant differences in the accuracy of the 6 IOL power calculation formulas in eyes targeted for myopia, further investigation into methods ideal for targeting myopia is necessary.

Despite being a retrospective study, this study had several strengths. First, eyes with a single type of IOL were included to eliminate differences in the refractive outcome due to variations in the IOL design. ${ }^{14,15}$ Second, the target refraction of intentional myopia was limited within the acceptable range (between $-2.0 \mathrm{D}$ and $-3.0 \mathrm{D}$ ). ${ }^{1,2}$ Third, only eyes with mild axial myopia were included, as high myopia could worsen the refractive outcome. ${ }^{7}$ Patients with high myopia were evaluated separately in the secondary analyses; we found a relatively myopic trend in the refractive outcomes for eyes with targeted intentional myopia as compared with emmetropia. This study also had some limitations, including small sample size, multiple surgeons, and a short follow-up period. Future prospective studies involving a large number of patients with a longer follow-up period are required to validate the results of this study.

Even though we found no significant difference in the ocular biometric parameters between the two groups, we did observe a significant difference in the refractive outcomes between eyes with a target of emmetropia and intentional myopia using some of the IOL power calculation formulas. It is likely that the requirement of an IOL with a higher power for targeting myopia may have enhanced the effect of any inaccuracy in the calculation process on predicting postoperative refraction. ${ }^{4}$ IOL power calculation formulas are presumed to have been developed for achieving emmetropia. Thus far, the mechanisms through which the examined formulas calculate refractive predictions for intentional myopia have not been well understood. Our results suggest that we may need to develop more optimal IOL power calculation methods for targeting myopia.

\section{Conclusion}

Some IOL power calculation formulas including SRK/T, Holladay 1, Hoffer Q, and Holladay 2 show a relative myopic trend in the refractive outcome when targeting intentional myopia as compared to emmetropia. Newer generation formulas such as Haigis and Barrett Universal II might be less affected by targeting intentional myopia.

\section{Abbreviations}

$\mathrm{AL}$, axial length; IOL, intraocular lens; SRK/T, Sanders, Retzlaff, and Kraft/theoretical.

\section{Data Sharing Statement}

All data included in this study are available from the corresponding author upon reasonable request.

\section{Ethics Approval and Informed Consent}

This retrospective study was performed in accordance with the Declaration of Helsinki and was approved by the Medical Ethics Committee of the Kobe City Medical Center General Hospital (Kobe, Japan). We applied an optout arrangement to obtain the informed consent for this observational study, which involved the analysis of medical records. The confidentiality of patient data was maintained.

\section{Acknowledgments}

We thank Mika Watanabe (Kobe City Eye Hospital) for collecting data.

\section{Author Contributions}

All authors made a significant contribution to the work reported, whether that is in the conception, study design, 
execution, acquisition of data, analysis and interpretation, or in all these areas; took part in drafting, revising or critically reviewing the article; gave final approval of the version to be published; have agreed on the journal to which the article has been submitted; and agree to be accountable for all aspects of the work.

\section{Funding}

There is no funding to report.

\section{Disclosure}

The authors declare no conflicts of interest.

\section{References}

1. Kora Y, Yaguchi S, Inatomi M, Ozawa T. Preferred postoperative refraction after cataract surgery for high myopia. $J$ Cataract Refract Surg. 1995;21(1):35-38. doi:10.1016/s0886-3350(13)80476-2

2. Hayashi K, Hayashi H. Optimum target refraction for highly and moderately myopic patients after monofocal intraocular lens implantation. $J$ Cataract Refract Surg. 2007;33(2):240-246. doi:10.1016/j.jcrs.2006.10.045

3. Dalto RF, Ferreira MA, Queiroz W, Coelho RP, Paula JS, Messias A. Haigis and SRKT formulae accuracy for intentional myopic overcorrection. Int Ophthalmol. 2018;38(4):1459-1463. doi:10.1007/ s10792-017-0607-2

4. Turnbull AMJ, Hill WE, Barrett GD. Accuracy of intraocular lens power calculation methods when targeting low myopia in monovision. $J$ Cataract Refract Surg. 2020;46(6):862-866. doi:10.1097/j.jcrs. 0000000000000187

5. Armstrong RA. Statistical guidelines for the analysis of data obtained from one or both eyes. Ophthalmic Physiol Opt. 2013;33(1):7-14. doi:10.1111/opo.12009
6. Simpson MJ, Charman WN. The effect of testing distance on intraocular lens power calculation. J Refract Surg. 2014;30(11):726.

7. Haigis W. Intraocular lens calculation in extreme myopia. $J$ Cataract Refract Surg. 2009;35(5):906-911. doi:10.1016/j.jcrs.2008.12.035

8. Behndig A, Montan P, Stenevi U, Kugelberg M, Zetterström C, Lundström M. Aiming for emmetropia after cataract surgery: Swedish National Cataract Register study. J Cataract Refract Surg. 2012;38(7):1181-1186. doi:10.1016/j.jcrs.2012.02.035

9. Yokoi T, Moriyama M, Hayashi K, Shimada N, Ohno-Matsui K. Evaluation of refractive error after cataract surgery in highly myopic eyes. Int Ophthalmol. 2013;33(4):343-348. doi:10.1007/s10792-0129690-6

10. Morgan IG, French AN, Ashby RS, et al. The epidemics of myopia: aetiology and prevention. Prog Retin Eye Res. 2018;62:134-149. doi:10.1016/j.preteyeres.2017.09.004

11. Nakao S, Miyake M, Hosoda Y, et al. Myopia prevalence and ocular biometry features in a general Japanese population: the Nagahama study. Ophthalmology. 2020;128(4):522-531. doi:10.1016/j.oph tha.2020.08.023

12. Geggel HS. Comparison of formulas and methods for high myopia patients requiring intraocular lens powers less than six diopters. Int Ophthalmol. 2018;38(4):1497-1504. doi:10.1007/s10792-0170611-6

13. Cooke DL, Huie T, Pletcher J. IOL power formula accuracy for intentional myopic overcorrection. J Cataract Refract Surg. 2021;47 (9):1237-1238. doi:10.1097/j.jcrs.0000000000000456

14. MacLaren RE, Natkunarajah M, Riaz Y, Bourne RRA, Restori M, Allan BDS. Biometry and formula accuracy with intraocular lenses used for cataract surgery in extreme hyperopia. Am J Ophthalmol. 2007;143(6):920-931. doi:10.1016/j.ajo.2007.02.043

15. Savini G, Barboni P, Ducoli P, Borrelli E, Hoffer KJ. Influence of intraocular lens haptic design on refractive error. $J$ Cataract Refract Surg. 2014;40(9):1473-1478. doi:10.1016/j.jcrs.2013.12.018
Clinical Ophthalmology

\section{Publish your work in this journal}

Clinical Ophthalmology is an international, peer-reviewed journal covering all subspecialties within ophthalmology. Key topics include: Optometry; Visual science; Pharmacology and drug therapy in eye diseases; Basic Sciences; Primary and Secondary eye care; Patient Safety and Quality of Care Improvements. This journal is indexed on PubMed

\section{Dovepress}

Central and CAS, and is the official journal of The Society of Clinical Ophthalmology (SCO). The manuscript management system is completely online and includes a very quick and fair peer-review system, which is all easy to use. Visit http://www.dovepress.com/ testimonials.php to read real quotes from published authors. 